Gesundheitsförderung Schweiz Promotion Santé Suisse Promozione Salute Svizzera

\section{Standpunkt}

\section{HausärztInnen als Bewegungsberater}

Der Hausärztin, dem Hausarzt kommt eine wichtige Rolle in der Gesundheitsberatung zu. Denn das Vertrauen der PatientInnen in ihre/seine Kompetenz ist ungetrübt - zumindest was die Beratung für einen gesunden Lebensstil angeht. Dies sollten wir ausnutzen. Häufig ist den PatientInnen eigentlich klar, dass Bewegungsarmut, Übergewicht und Rauchen gesundheitsgefährdend sind und sie scheinen froh, darauf angesprochen zu werden. Dies ist auch die Erfahrung der ersten Befragungswochen des Anfang Jahr gestarteten Pilotprojekts „Gesund bewegt“, das sich mit Bewegungsförderung befasst: PatientInnen schätzen die Initiative der ÄrztInnen. Und es entstehen oft schon in der Sprechstunde Gespräche über das Vorbeugen von Krankheiten, das Fördern der Gesundheit oder die steigenden Kosten im Gesundheitswesen. „Gesund bewegt" wird in der Nordwestschweiz durchgeführt und baut auf dem Projekt „Bewegung statt Verkehr ÄrztInnen beraten“ auf, das an der „sun 21“ mit dem Hauptpreis 2003 ausgezeichnet wurde. Mehrmals während zweier Wochen werden PatientInnen aus 30 Arztpraxen im Alter von 16 bis 65 Jahren mittels Fragebogen vor der Arztkonsultation befragt und in der Sprechstunde als körperlich aktiv oder inaktiv eingestuft. In der ersten Woche erhalten alle Inaktiven eine Informationsbroschüre über Bewegung im Alltag. In der zweiten Woche bekommen die Inaktiven einen Gutschein für eine Bewegungsberatung bei einem ausgebildeten Bewegungsberater, sei dies ein(e) ÄrztIn oder ein(e) PhysiotherapeutIn. So wird der Effekt einer Beratung mit der Abgabe der Broschüre verglichen. Die Ausbildung der BeraterInnen wird mit dem Bundesamt für Sport organisiert. Die Teilnehmenden arbeiten eine Online-Ausbildung (siehe www.ratzurtat.ch) durch und schliessen diese mit einem ganztägigen Workshop ab.

Schon „Vom Rat zur Tat“, ein 2000 abgeschlossenes Projekt des Instituts für Sozial- und Präventivmedizin Zürich und des Bundesamts für Sport (BASPO), in dem ÄrztInnen die Beratung an BewegungsberaterInnen delegierten, hatte festgestellt, dass allein die Befragung durch den Hausarzt beim Patient oder der Patientin etwas bewirkt. Diese erstaunliche Tatsache, bewog uns, den Ansatz weiter zu ver-

folgen und die Erfahrungen auf breiterer Basis zu dokumentieren und zu evaluieren. Durch gezielte Informationen oder durch Beratung könnten ÄrztInnen vielleicht noch mehr Leute zu mehr Bewegung motivieren!

Das bisherige Echo auf unser Projekt ist positiv. Das Projekt dürfte sich insbesondere auf die Prophylaxe von HerzKreislaufkrankheiten, Diabetes, Adipositas und auf das Wohlbefinden auswirken. Daneben erreichen wir eine Reduktion des motorisierten Verkehrs, verbunden mit einer Schadstoff- und Lärmreduktion. Dies ist eine Hintergrundabsicht des Projekts, das von den Ärztinnen und Ärzten für Umweltschutz beider Basel entwickelt wurde und das neben Gesundheitsförderung Schweiz auch von den Umweltämtern der Kantone Basel-Stadt und Baselland von mehreren Bundesämtern unterstützt wird.

Max Handschin ist Hausarzt in Gelterkinden BL. Er ist Vorstandsmitglied der Ärztinnen und Ärzte für Umweltschutz. beider Basel und beteiligt am Projekt „Gesund bewegt“, das er mitentwickelt hat.

Mehr zum Thema Bewegungsberatung in der Hausarztpraxis im Internet unter: www.gesundbewegt.ch und www.baspo.ch (siehe Wissenschaft/ Gesundheitsförderung/Interventionen und Evaluation).

\title{
Echo der Praxis
}

\section{Projekte mit Kantonen}

Gesundheitsförderung Schweiz unterstützt unter anderem Projekte, die mit den Kantonen geplant und umgesetzt werden. Zum Beispiel „50+santé - Gesundheitsförderung für Menschen über 50“, ein interkantonales Projekt, an dem sich alle sieben Kantone der lateinischen Schweiz beteiligen. Das Projekt, für das sich auch die Westschweizer Konferenz für Gesundheitswesen und soziale Angelegenheiten (CRASS) stark macht, setzt bei den Lebens- und Arbeitsumständen von älteren Menschen an. Es ist am 1. Juli 2004 gestartet; seine Laufzeit ist vorderhand auf drei Jahre beschränkt. Eine erste Gruppe von Pilotprojekten wurde im Oktober 04 ausgewählt. Eine zweite Gruppe wird im März 05 bestimmt.

Projekt-Newsletter erhältlich bei vera.sandoval@adsan.org. Mehr zur Förderung von Projekten mit Kantonen im Internet unter: www.gesundheitsfoerderung.ch.

Auf dieser Seite erscheinen Informationen von und über Gesundheitsförderung Schweiz. Die Stiftung hat zum Ziel, die Gesundheitsressourcen aller in der Schweiz lebenden Menschen zu fördern. Im Auftrag von Bund, Kantonen und Krankenkassen unterstützt sie Projekte, welche die Gesundheit stärken und koordiniert solche Aktivitäten gemeinsam mit Partnern.

Dufourstr. 30, Postfach 311 CH-3000 Bern 6

Tel. +41 (0) 313500404 Fax +41 (0) 313681700 office.bern@promotionsante.ch www.gesundheitsfoerderung.ch
Avenue de la Gare 52, Case postale 670 CH-1001 Lausanne Tel. +41 (0) 213451515 Fax +41 (0) 213451545 office@promotionsante.ch www.promotionsante.ch 